\title{
A retrospective multicenter study comparing bupivacaine and ropivacaine in endoscopic ultrasound guided celiac plexus neurolysis
}

\author{
Yuchong Zhao" ${ }^{1 \#}$ Xiaorong Guo ${ }^{2 \#}$, Kaixuan Wang ${ }^{2}$, Qian Chen ${ }^{1}$, Yun Wang ${ }^{1}$, Liangkai Chen ${ }^{3}$, Wei Gao ${ }^{4}$, \\ Bin Cheng ${ }^{1} \wedge$, Zhendong Jin ${ }^{2}$
}

${ }^{1}$ Department of Gastroenterology and Hepatology, Tongji Hospital, Tongji Medical College, Huazhong University of Science and Technology (HUST), Wuhan, China; ${ }^{2}$ Department of Gastroenterology and Hepatology, Changhai Hospital, The Secondary Military Medical University, Shanghai, China; ${ }^{3}$ Department of Epidemiology and Biostatistics, School of Public Health, Tongji Medical College, HUST, Wuhan, China; ${ }^{4}$ Department of Anesthesia, Xiangya Hospital, Central South University, Changsha, China

Contributions: (I) Conception and design: B Cheng, Z Jin; (II) Administrative support: B Cheng, Z Jin, K Wang, Q Chen; (III) Provision of study materials or patients: None; (IV) Collection and assembly of data: Y Zhao, X Guo; (V) Data analysis and interpretation: Y Zhao, X Guo, L Chen; (VI) Manuscript writing: All authors; (VII) Final approval of manuscript: All authors.

"These authors contributed equally to this work.

Correspondence to: Bin Cheng, MD. Department of Gastroenterology and Hepatology, Tongji Hospital, Tongji Medical College, Huazhong University of Science and Technology (HUST), Wuhan, China. Email: b.cheng@tjh.tjmu.edu.cn; Zhendong Jin, MD. Department of Gastroenterology and Hepatology, Changhai Hospital, The Secondary Military Medical University, Shanghai, China. Email: zhendjin@163.com.

Backgroundz Endoscopic ultrasound-guided celiac plexus neurolysis (EUS-CPN) is widely practiced to palliate cancer pain in patients with inoperable abdominal malignancy. During CPN, the dehydrated alcohol is injected to ablate neural tissue and local anesthetics is to minimize the discomfort from alcohol injection. This study aims to compare the efficacy and safety of bupivacaine and ropivacaine in EUS-CPN.

Methods: We retrospectively pooled and analyzed two large EUS centers' 150 consecutive patients underwent EUS-CPN from January 2012 to March 2019. Patients were divided into 3 groups based on the selection of anesthetics: $0.5 \%$ ropivacaine (ROPI, $n=23$ ), $0.375 \%$ bupivacaine $(0.375 \%$ BUPI, $n=21), 0.75 \%$ bupivacaine (0.75\% BUPI, $\mathrm{n}=106)$. Visual analogue scale (VAS) was used to measure pre/post-operative pain at 11 observation points. Additional data were collected from medical records.

Results: The incidence of procedure-related pain within 12 hours after CPN was significantly different among the three groups, $10.38 \%$ in $0.75 \%$ BUPI [OR =0.26 (95\% CI: 0.07-0.94); P=0.04], 26.09\% in ROPI and $23.81 \%$ in $0.375 \%$ BUPI group, respectively. The risk of post-procedural arrhythmia was similar between the $0.375 \%$ and $0.75 \%$ BUPI groups (19.05\% versus $18.87 \%$ ), while relatively lower in ROPI group (13.04\%). No patients in any group developed symptoms of CNS toxicity related to anesthetics.

Conclusions: Compared with $0.375 \%$ bupivacaine and $0.5 \%$ ropivacaine, $0.75 \%$ bupivacaine in CPN can reduce post-procedural pain. Ropivacaine shows a tendency of less arrhythmogenic effect in CPN.

Keywords: Cancer pain; endoscopic ultrasound; celiac plexus neurolysis (CPN); ropivacaine; bupivacaine

Submitted May 22, 2020. Accepted for publication Aug 10, 2020.

doi: 10.21037/apm-20-1158

View this article at: http://dx.doi.org/10.21037/apm-20-1158

^ ORCID: 0000-0003-1568-8760. 


\section{Introduction}

Patients with pancreatic cancer have a poor prognosis and they often suffer from intractable cancer-related pain. Endoscopic ultrasound-guided celiac plexus neurolysis (EUS-CPN) is a safe and effective modality for pain management, especially in patients with opioid narcotics resistance (1). EUS-CPN directly accesses the celiac plexus, avoiding the spinal nerves, arteries or diaphragm (1). Compared to percutaneous CPN, EUS-CPN appears to be less likely to cause serious complications, such as accidental lumbar puncture, paresthesia, and hemopneumothorax (2). Moreover, EUS guided fine needle aspiration (FNA) can be accomplished during the same session for tumor staging if needed.

During CPN, dehydrated alcohol (>98\% ethanol) is used to ablate neurons for pain relief. However, alcohol injection alone may cause pain because of perineurium swelling and tissue damage. Additionally, its analgesia takes a few days to achieve maximal effect after EUSCPN. During conscious sedation, the patients can present with obvious discomfort after anesthetics injection when bupivacaine or ropivacaine is not utilized initially $(1,2)$. Acute post-procedural pain leads to a series of pathophysiological and behavioral responses, resulting in deleterious stress responses including arrhythmia, hypertension, immunosuppression, and stasis (3). In contrast, appropriate analgesia and pain management will improve procedural outcomes with reduced hospitalstay time and morbidity (4). The endoscopists performing EUS-CPN often concern about the choice of anesthetic agents and their optimal concentration. Three to $10 \mathrm{~mL}$ $0.25-0.75 \%$ bupivacaine is customarily used, however, ropivacaine offers an option as a new long-acting amide local anesthetic, which has less central nervous system and cardiovascular toxicities than bupivacaine. Ropivacaine has been widely used during peri-nerve block and labor analgesia (5-7). Furthermore, bupivacaine is relatively more potent than other amide derivatives anesthetics (5). Many patients still can develop post-procedural discomfort within hours. The incidence of transient pain exacerbation was reported to be $8.6-29.4 \%$ (8-10). We conducted this multicenter retrospective study to compare ropivacaine and bupivacaine at different concentrations during EUSCPN. We present the following article in accordance with the STROBE reporting checklist (available at http://dx.doi. org/10.21037/apm-20-1158).

\section{Methods}

\section{Patients}

This retrospective study was conducted at 2 of the largest EUS centers in China (Tongji Hospital of Tongji Medical College and Changhai Hospital of The Secondary Military Medical University) and approved by the Ethical Committee of Tongji Hospital, Tongji Medical College, Huazhong University of Science and Technology (TJ-IRB20190906) and was conducted according to the principles of the Declaration of Helsinki (as revised in 2013). Thus, this is a retrospective study, we apply for exemption of informed consent. One hundred fifty consecutive cases of EUS-CPN were reviewed retrospectively from January 2012 to March 2019. The patient's data were considered eligible when they met inclusion criteria: age $\geq 18$, diagnosis of inoperable upper abdominal cancer, and VAS $\geq 4$ after the use of opioid narcotics. Only patients in whom $0.5 \%$ ropivacaine (ROPI group), $0.375 \%$ bupivacaine (0.375 BUPI group) or $0.75 \%$ bupivacaine was used, were included in the final analysis of the data. Patients with incomplete data ( $\geq 2$-time postoperative VAS scoring or ECG missing) were excluded from the analysis.

\section{Measurement}

As a part of routine clinical practice in these two EUS centers, pain assessment was made with standard 11-point VAS with "0" equaling no pain and "10" worst pain under the instruction by a physician (other than the endoscopist who performed EUS), and a registered nurse would assist the patient to fulfill the assessment $(11,12)$. All enrolled patients were offered VAS pain assessment at preCPN 2 days, 1 day, 3 hours, and post-CPN 2, 4, 6, 8, 10, 24 hours $( \pm 30 \mathrm{~min})$, VRS (verbal rating scale) pain assessment at 48, 72 hours postoperative. Electrocardiogram (ECG) monitoring was performed during EUS-CPN and one hour afterward, another ECG was performed before discharge. Procedure-related pain was defined as postoperative acute pain exacerbation due to dehydrated alcohol injection-induced plexus perineurium swelling and tissue damage, which last no longer than 4 days (median 24 hours) $(1,2,13)$. This discomfort transiently got worse than preoperative pain, and patients were generally in requirement for rescue dose narcotics including NSAIDS, gabapentin, pregabalin, even morphine, and fentanyl 

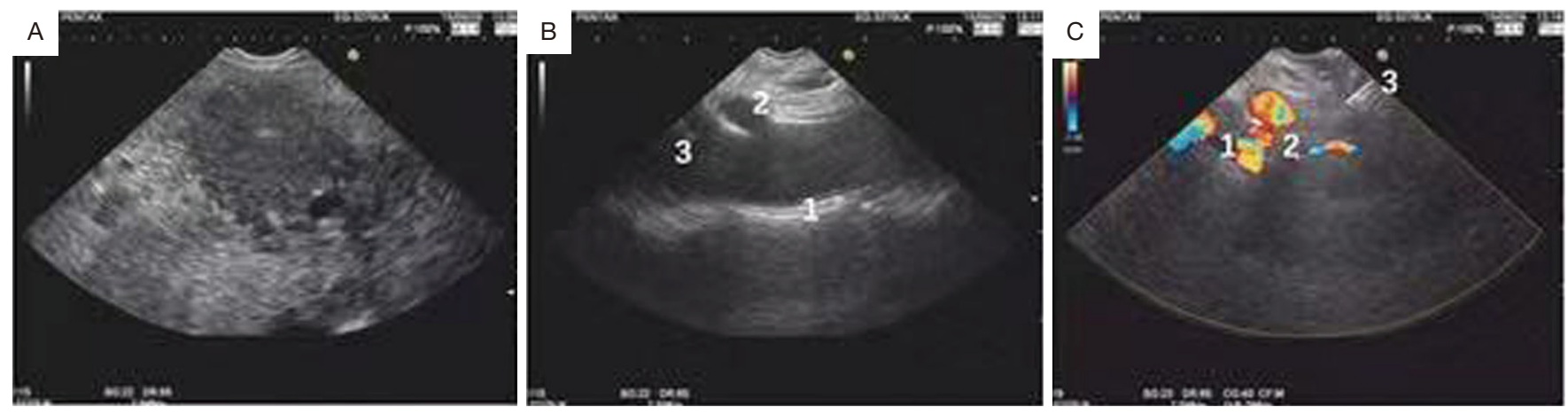

Figure 1 Endoscopic ultrasonography-guided celiac plexus neurolysis imaging. (A) Pancreatic solid mass. (B) 1, abdominal aorta; 2, celiac trunk; 3, superior mesenteric artery. (C) 1, abdominal aorta; 2, celiac trunk; 3, aspiration needle.

$(14,15)$. Patients meet criteria: (I) VAS score within 6 hours after CPN greater than preoperative score; (II) VAS score after 24 hours less than preoperative score; (III) ruling out other diseases leading to pain exacerbation were recognized cases with postoperative procedurerelated pain. Time-varying narcotic medication including opiates kinds and dosage was documented from pre-CPN 1 week to 1 week after the procedure. To standardize pain palliation medication usage, the dose was converted to an equianalgesic dosage $(\mathrm{mg} /$ day) of orally administrated morphine according to established standards (8).

\section{Technique}

An anesthetist applied intravenous propofol for sedation according to the principles of "monitored anesthesia care". Patients uniformly took the left lateral decubitus position during the procedure. All patients received oxygen during the procedure and blood pressure and heart rate were monitored. When EUS-FNA was performed, the smears were then immediately reviewed by a cytopathologist. After staging and FNA cytological diagnosis, patients with unresectable cancer underwent EUS-CPN. EUS-CPN was performed with a linear array echoendoscope (GF-UCT260, Olympus Inc., Melville, NY, USA). Briefly, sagittal views of the aorta were obtained at the conjunction of gastric fundus and body of the lesser curvature. Afterward, the aorta was traced to the celiac trunk and superior mesenteric artery by revolving the echoendoscope anticlockwise (Figure 1). Under direct EUS visualization, a 22 to 23 gauge, 4 to $8 \mathrm{~cm}$ aspiration needle (Wilson-Cook Medical, Inc. Winston-
Salem, NC, USA) primed with normal saline solution was placed immediately adjacent and anterior to the lateral aspect of the aorta at the level of the celiac trunk. After injecting $2 \mathrm{~mL}$ of saline solution to clear the needle, an aspiration test was performed. If no blood was obtained, $5 \mathrm{~mL}$ of $0.5 \%$ ropivacaine, $0.375 \%$ bupivacaine or $0.75 \%$ preservative-free bupivacaine was injected according to the operator's prior habit. The aspiration test was repeated, and if there was no blood return, $10 \mathrm{~mL}$ of dehydrated $98 \%$ absolute alcohol was injected. The needle was then flushed with $3 \mathrm{~mL}$ of saline solution and withdrawn from the patient. Endosonographically, an echo-dense cloud was typically identified with alcohol injection. The process was then repeated on the opposite side of the aorta. The average estimated time for the EUS-CPN portion of the procedure was 15 minutes.

\section{Statistical analysis}

The characteristics of patients were summarized as mean \pm standard deviation (SD) for normally distributed continuous variables, median with interquartile range for continuous variables with skewed distribution, and frequency (percentage) for categorical variables. We performed multivariable logistic regression analysis to estimate the independent association between pain scores/ postoperative arrhythmia and the risk of anesthetics with an adjustment for potential confounders. All of the analyses were performed using R (http://www.R-project. org, version 3.5.2) with a two-sided significance threshold of $\mathrm{P}<0.05$. 
Table 1 Baseline characteristics of all patients

\begin{tabular}{|c|c|c|c|c|}
\hline Characteristics & ROPI (n=23) & $0.375 \%$ BUPI $(n=21)$ & $0.75 \%$ BUPI $(n=106)$ & $P$ value \\
\hline Male, n (\%) & $10(43.48)$ & $12(57.14)$ & 79 (74.53) & 0.009 \\
\hline \multicolumn{5}{|l|}{ Type of malignancy, n (\%) } \\
\hline Pancreatic cancer & & & & 0.159 \\
\hline Body & $2(8.70)$ & $1(4.76)$ & $28(26.42)$ & \\
\hline Tail & $5(21.74)$ & $4(19.05)$ & $13(12.26)$ & \\
\hline Diffuse involvement & $2(8.70)$ & $5(23.81)$ & $16(15.09)$ & \\
\hline Other & $6(26.09)$ & $4(19.05)$ & $2(1.89)$ & $<0.001$ \\
\hline Prior narcotic use, $n(\%)$ & $15(65.22)$ & $10(47.62)$ & 52 (49.06) & 0.348 \\
\hline Pre-CPN arrhythmia, n (\%) & $1(4.35)$ & $3(14.29)$ & $11(10.38)$ & 0.569 \\
\hline
\end{tabular}

Others including hepatocellular cancer, gastric cancer, colonic cancer and cholangiocellular carcinoma. BUPI, bupivacaine; ROPI, ropivacaine; CPN, celiac plexus neurolysis.

\section{Results}

\section{Patient characteristics}

In this study, 23 patients received CPN with $0.5 \%$ Ropivacaine, 21 with $0.375 \%$ Bupivacaine, and 106 patients with $0.75 \%$ Bupivacaine. Of the entire cohort, there were 101 men and 49 women (mean age 63-year-old; range 37 to 87 years old). The baselines of patient characteristics are shown in Table 1. None of the patients accepted pre-CPN adjuvant treatment including chemotherapy or radiation within 2 weeks.

\section{Analysis of efficacy}

Pain scores decline gradually within 3 days after EUS-CPN (Figure 2). Among all 150 patients, we observed 6 patients in the ROPI group with the transient pain exacerbation related to CPN procedure (26.09\%), 5 (23.81\%) in $0.375 \%$ BUPI group, and $11(10.38 \%)$ in $0.75 \%$ BUPI group [OR $=0.26$ (95\% CI: 0.07-0.94); $\mathrm{P}=0.04]$, respectively. In addition, 1 patient in the ROPI group had intermittent pain due to typical enterospasm, which was not identified as the procedure-related pain and the symptoms disappeared after treatment of cimetropium bromide. Furthermore, 2 of the 5 in $0.375 \%$ BUPI group and 1 of the 11 in $0.75 \%$
BUPI group had EUS-FNA simultaneously. Overall, the incidence of postoperative pain in $0.75 \%$ BUPI group was significantly lower, while no significant difference was noted between $0.37 \%$ BUPI and $0.5 \%$ ROPI groups (Table 2).

\section{Complications}

There were no major complications during or after the procedures. Two patients had hematemesis after the procedure, and alcohol-related agitation and ataxia were observed in one patient. One patient had transient hypotension $(55 / 25 \mathrm{mmHg})$ and recover within 6 hours. No obvious CNS toxicity concerned with analgesics was noted.

Sinus tachycardia was the most common post-procedure arrhythmia, others consist of sinus bradycardia, premature ventricular contraction, premature atrial contraction, ventricular tachycardia and atrial fibrillation (Table 3). There was no statistical difference among the incidence of arrhythmia of the 3 groups (Table 4). One patient with normal pre-procedure ECG in $0.375 \%$ BUPI group developed the post-procedure atrial fibrillation and frequent multisource premature ventricular contraction and thus went through cardioversion by cedilanid treatment. None of the other arrhythmia patients had hemodynamic disorder according to the ECG monitoring. 


\section{Discussion}

EUS-CPN has been established as a safe and effective modality for pain palliation in patients with inoperable upper abdominal cancers (1,9,16-18). A novel machine learning approach can precisely identify whether those subjects prone to benefit from repeat CPN (10). During the procedure, anesthetics are used to palliate discomfort caused by dehydrated alcohol injection but because the palliative effects gradually reach the peak and therefore the postoperative pain could not be completely avoided after EUS-CPN (1). Nevertheless, the analgesic options for EUS$\mathrm{CPN}$ procedure are limited since the pain exacerbation

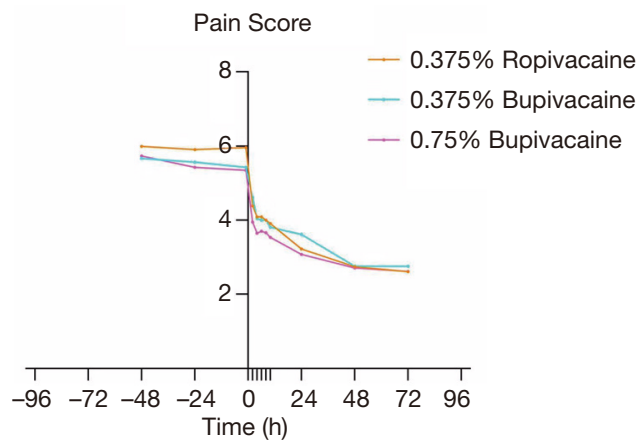

Figure 2 Pre- and post-procedure mean pain scores over time. after EUS-CPN is transient and usually does not last long compared to those ongoing postoperative pain of thoracic, abdominal or lower extremity surgeries. For example, the postoperative epidural opioid or epidural local anesthetics are considered not necessary and economic. NSAIDs are the usual option for postoperative pain relief with an antiinflammatory effect $(19,20)$, whereas the potential relevant side-effects of NSAIDs contain gastrointestinal irritation, platelet inhibition, and renal insufficiency. Given the lack of management of this complication, we thus studied the alternative analgesics for the procedure.

In reality, the choices of anesthetics and its concentration are usually made according to endoscopists' experience and preference. Bupivacaine and ropivacaine both belong to the long-acting amino-amide local anesthetics through inhibiting voltage-gated sodium channels in nerve fibers (5). Bupivacaine of concentration from $0.25 \%$ to $0.75 \%$ is the conventional choice in CPN procedure as a powerful and inexpensive local anesthetics, which has approximately $50 \%$ higher analgesia value than ropivacaine of the same concentration $(1,2,5,8,9,21)$. Due to the relatively lower cardiovascular and CNS toxicity, ropivacaine is less lipophilic and is used widely as a substitute due to better tolerance, especially for labor analgesia $(6,7)$. Another advantage of ropivacaine is minor motor conduction block compared with bupivacaine in peripheral nerve

Table 2 Comparison of incidence of postoperative pain among 3 groups

\begin{tabular}{|c|c|c|c|c|c|c|}
\hline Model & \multicolumn{2}{|c|}{$0.375 \%$ BUPI } & \multicolumn{2}{|c|}{ ROPI } & \multicolumn{2}{|c|}{ 0.75\% BUPI } \\
\hline Crude model & 1.00 (ref.) & - & $0.69(0.18,2.73)$ & 0.602 & $0.26(0.08,0.82)$ & 0.022 \\
\hline Model 1 & 1.00 (ref.) & - & $0.75(0.19,3.03)$ & 0.689 & $0.26(0.08,0.86)$ & 0.027 \\
\hline Model 2 & 1.00 (ref.) & - & $1.05(0.24,4.60)$ & 0.951 & $0.26(0.07,0.94)$ & 0.040 \\
\hline
\end{tabular}

Statistical analysis are based on multivariable logistic regression model. Model 1 was adjusted for aged and sex. Model 2 was additionally adjusted for pre-procedure VAS score, pre-procedure narcotics use, direct invasion of celiac plexus, location of the tumor. BUPI, bupivacaine; ROPI, ropivacaine; OR, odds ratio.

Table 3 Cases of postoperative arrhythmia

\begin{tabular}{|c|c|c|c|c|c|c|c|c|}
\hline Group & SB & ST & PVC & PAC & VT & $\mathrm{AF}$ & QRS duration prolong & Overall patients \\
\hline $0.375 \%$ BUPI, n & - & 1 & 2 & 1 & - & 1 & 0 & 4 \\
\hline $0.75 \%$ BUPI, $n$ & 2 & 15 & - & - & - & 2 & 2 & 20 \\
\hline
\end{tabular}

SB, sinus bradycardia; ST, sinus tachycardia; PVC, premature ventricular contraction; PAC, premature atrial contraction; VT, ventricular tachycardia; AF, atrial fibrillation; BUPI, bupivacaine; ROPI, ropivacaine. 
Table 4 Comparison of incidence of postoperative arrhythmia among 3 groups

\begin{tabular}{|c|c|c|c|c|c|c|}
\hline Model & \multicolumn{2}{|c|}{$0.375 \%$ BUPI } & \multicolumn{2}{|l|}{ ROPI } & \multicolumn{2}{|c|}{$0.75 \%$ BUPI } \\
\hline Crude model & 1.00 (ref.) & - & $0.64(0.12,3.26)$ & 0.588 & $0.99(0.30,3.26)$ & 0.985 \\
\hline Model 1 & 1.00 (ref.) & - & $0.70(0.13,3.70)$ & 0.679 & $0.85(0.25,2.90)$ & 0.799 \\
\hline Model 2 & 1.00 (ref.) & - & $0.89(0.16,5.06)$ & 0.898 & $0.95(0.26,3.46)$ & 0.943 \\
\hline
\end{tabular}

Statistical analysis are based on multivariable logistic regression model. Model 1 was adjusted for age and sex. Model 2 was additionally adjusted for pre-procedure ECG. ECG, electrocardiogram; BUPI, bupivacaine; ROPI, ropivacaine; OR, odds ratio.

blocks $(22,23)$, though this is not our first consideration in CPN. Analgesia effect of ropivacaine could last 3-6 hours or longer, while levobupivacaine produces an estimated $30 \%$ longer duration of ultimate plexus block than ropivacaine $(5,24)$. Generally, patients in all 3 study groups had sufficient pain relief compared to preoperative situations. Our study demonstrates $0.75 \%$ bupivacaine has a significantly better analgesic effect for postoperative pain than $0.375 \%$ bupivacaine and $0.5 \%$ ropivacaine, though differences between bupivacaine and ropivacaine of the same concentration were not significant. Patients with CPN procedures were discharged within 24 hours if no complications occurred. The reduction of postoperative pain would contribute to better recovery and length of hospital stay (25).

Another important consideration factor of choosing among different anesthetics is the complication risk. As previously reported by a few clinical studies, bupivacaine had increased CNS toxicity than ropivacaine in humans and animals by intravenous administration, and ropivacaine required a $10-25 \%$ larger dose than bupivacaine before CNS intoxication occurred $(5,6)$. Although CNS intoxication, from shivering to convulsion, is usually more frequent and evident than cardiovascular effects, we did not observe typical CNS intoxication in our study. With regard to this result, CNS toxic effects of local anesthetics are much more difficult to evaluate in humans than in animals. As for cardiovascular intoxication, bupivacaine and ropivacaine both show dose-dependent cardiac toxicity with persistently increased PR interval and prolonged QRS duration. Nonetheless, bupivacaine-induced potent conduction block can be accumulated and the lethal dose of bupivacaine and ropivacaine are usually $2: 1$ in animals $(5,24)$. Most arrhythmia recorded in Holter monitoring was transient without any severe consequences. Despite the difference of arrhythmia was not significant in our study, we still could see a tendency of reduced potential in cardiovascular toxicity in comparison of $0.375 \%$ bupivacaine and $0.75 \%$ bupivacaine through odds ratio. A reasonable explanation for this result is the unbalanced and inadequate sample among groups in virtue of our study set in a realworld model. Another conceivable reason accounting for this is that bupivacaine or ropivacaine in $\mathrm{CPN}$ is not intravenous administrated and relatively low-dose of $5 \mathrm{~mL}$ or less. Thus, we still incline to the view that ropivacaine has reduced potential for cardiovascular intoxication. However, we cannot attribute the occurrence of arrhythmia to anesthetics use. Most arrhythmia of our patients still could be recorded after $24 \mathrm{~h}$, theoretically, bupivacaine and ropivacaine have superseded for a long time. In peripheral nerve block, arrhythmia was rarely reported $(5,22)$. And most of our patients who accepted CPN were aged, infirm with existing physical illness. Hence, we tend to consider postoperative arrhythmia caused by multi factors including anesthetics, celiac plexus destruction, etc.

Taken both efficacy and safety into consideration, we recommend that $0.75 \%$ bupivacaine should be considered as a protective agent for postoperative procedure-related pain when patients do not have cardiovascular diseases. Ropivacaine shows significantly reduced toxic effects compared with bupivacaine in continued epidural analgesia, spinal anesthesia. However, the anesthesia effect of lowdose/concentration ropivacaine in $\mathrm{CPN}$ is not satisfying enough. With relatively more patients in $0.75 \%$ bupivacaine group included, no severe cardiovascular toxic effects were observed. However, one patient had a hemodynamic disorder in $0.375 \%$ bupivacaine group, treated with cedilanid subsequently. In addition, CPN procedure itself could lead to transient hypotension owing to plexus destruction, it is more necessary to evaluate patients' cardiovascular conditions. Hence, we could not conclude currently that $0.75 \%$ bupivacaine is a better choice in CPN. Ropivacaine might be a corresponding protective agent to patients with past cardiovascular diseases. It is noteworthy 
that approximately $10 \%$ of patients still had postoperative pain when treated with $0.75 \%$ bupivacaine. The primary intention of our study is to raise endoscopists' concern about postoperative procedure-related pain which is seldom noticed before.

The major limitation of our study is the unequal sample capacity and a minority of patients in low-concentration groups, yet we imitate the clinical practice in a real-world model as possible. Further RCT interpreting differences between ropivacaine and bupivacaine is needed.

\section{Acknowledgments}

The authors wish to acknowledge all the anesthetists, nurses and physicians who have helped with pain assessment during the 8 years. Thanks for Dr. Shou-Jiang Tang from the University of Mississippi Medical Center for help with optimizing research advice.

Funding: This work was supported by National Natural Science Foundation of China Grants No. 81802427 (to ZL).

\section{Footnote}

Reporting Checklist: The authors have completed the STROBE reporting checklist. Available at http://dx.doi. org/10.21037/apm-20-1158

Data Sharing Statement: Available at http://dx.doi. org/10.21037/apm-20-1158

Peer Review File: Available at http://dx.doi.org/10.21037/ apm-20-1158

Conflicts of Interest: All authors have completed the ICMJE uniform disclosure form (available at http://dx.doi. org/10.21037/apm-20-1158). The authors have no conflicts of interest to declare.

Ethical Statement: The authors are accountable for all aspects of the work in ensuring that questions related to the accuracy or integrity of any part of the work are appropriately investigated and resolved. The study concerning the clients' right to privacy. The study was approved by the Ethical Committee of Tongji Hospital, Tongji Medical College, Huazhong University of Science and Technology (TJ-IRB20190906) and was conducted according to the principles of the Declaration of Helsinki (as revised in 2013). Thus, this is a retrospective study, we apply for exemption of informed consent.

Open Access Statement: This is an Open Access article distributed in accordance with the Creative Commons Attribution-NonCommercial-NoDerivs 4.0 International License (CC BY-NC-ND 4.0), which permits the noncommercial replication and distribution of the article with the strict proviso that no changes or edits are made and the original work is properly cited (including links to both the formal publication through the relevant DOI and the license). See: https://creativecommons.org/licenses/by-nc-nd/4.0/.

\section{References}

1. Harada N, Wiersema MJ, Wiersema LM. Endosonography-guided celiac plexus neurolysis. Gastrointest Endosc Clin N Am 1997;7:237-45.

2. Das A, Sivak MV Jr. Endoscopic palliation for inoperable pancreatic cancer. Cancer Control 2000;7:452-7.

3. Wu CL, Raja SN. Treatment of acute postoperative pain. Lancet 2011;377:2215-25.

4. Kehlet H, Holte K. Effect of postoperative analgesia on surgical outcome. Br J Anaesth 2001;87:62-72.

5. Casati A, Putzu M. Bupivacaine, levobupivacaine and ropivacaine: are they clinically different? Best Pract Res Clin Anaesthesiol 2005;19:247-68.

6. Scott DB, Lee A, Fagan D, et al. Acute toxicity of ropivacaine compared with that of bupivacaine. Anesth Analg 1989;69:563-9.

7. Knudsen K, Beckman Suurkula M, Blomberg S, et al. Central nervous and cardiovascular effects of i.v. infusions of ropivacaine, bupivacaine and placebo in volunteers. $\mathrm{Br} \mathrm{J}$ Anaesth 1997;78:507-14.

8. Gunaratnam NT, Sarma AV, Norton ID, et al. A prospective study of EUS-guided celiac plexus neurolysis for pancreatic cancer pain. Gastrointest Endosc 2001;54:316-24.

9. Doi S, Yasuda I, Kawakami H, et al. Endoscopic ultrasound-guided celiac ganglia neurolysis vs. celiac plexus neurolysis: a randomized multicenter trial. Endoscopy 2013;45:362-9.

10. Facciorusso A, Del Prete V, Antonino M, et al. Response to repeat echoendoscopic celiac plexus neurolysis in pancreatic cancer patients: A machine learning approach. Pancreatology 2019;19:866-72.

11. Collins SL, Moore RA, McQuay HJ. The visual analogue pain intensity scale: what is moderate pain in millimetres? 
Pain 1997;72:95-7.

12. Bijur PE, Silver W, Gallagher EJ. Reliability of the visual analog scale for measurement of acute pain. Acad Emerg Med 2001;8:1153-7.

13. Eloubeidi MA, Tamhane A. Prospective Assessment of Diagnostic Utility and Complications of Endoscopic Ultrasound-Guided Fine Needle Aspiration. Dig Dis 2008;26:356-63.

14. Sahgal N, Banerjee A. Efficacy of pregabalin in acute postoperative pain: a meta-analysis. Br J Anaesth 2011;107:274; author reply 275.

15. Mathiesen O, Moiniche S, Dahl JB. Gabapentin and postoperative pain: a qualitative and quantitative systematic review, with focus on procedure. BMC Anesthesiol 2007;7:6.

16. Kaufman M, Singh G, Das S, et al. Efficacy of Endoscopic Ultrasound-guided Celiac Plexus Block and Celiac Plexus Neurolysis for Managing Abdominal Pain Associated With Chronic Pancreatitis and Pancreatic Cancer. J Clin Gastroenterol 2010;44:127-34.

17. Sahai AV, Lemelin V, Lam E, et al. Central vs. Bilateral Endoscopic Ultrasound-Guided Celiac Plexus Block or Neurolysis: A Comparative Study of Short-Term Effectiveness. Am J Gastroenterol 2009;104:326-9.

18. Iwata K, Yasuda I, Enya M, et al. Predictive factors for pain relief after endoscopic ultrasound-guided celiac plexus neurolysis. Dig Endosc 2011;23:140-5.

Cite this article as: Zhao Y, Guo X, Wang K, Chen Q, Wang Y, Chen L, Gao W, Cheng B, Jin Z. A retrospective multicenter study comparing bupivacaine and ropivacaine in endoscopic ultrasound guided celiac plexus neurolysis. Ann Palliat Med 2021;10(2):1755-1762. doi: 10.21037/apm-20-1158
19. Chou R, Gordon DB, de Leon-Casasola OA, et al. Management of Postoperative Pain: A Clinical Practice Guideline From the American Pain Society, the American Society of Regional Anesthesia and Pain Medicine, and the American Society of Anesthesiologists' Committee on Regional Anesthesia, Executive Committee, and Administrative Council. J Pain 2016;17:131-57.

20. Hyllested M, Jones S, Pedersen JL, et al. Comparative effect of paracetamol, NSAIDs or their combination in postoperative pain management: a qualitative review. Br J Anaesth 2002;88:199-214.

21. Uchiyama Y, Hino S, Matsuda K, et al. Endosonographyguided celiac plexus neurolysis for cancer pain. Nihon Rinsho 2001;59 Suppl 4:621-5.

22. Fanelli G, Casati A, Beccaria P, et al. A double-blind comparison of ropivacaine, bupivacaine, and mepivacaine during sciatic and femoral nerve blockade. Anesthesia And Analgesia 1998;87:597-600.

23. Breuninger H, Nogova L, Hobbach PS, et al. Ropivacaine, an attractive agent for slow infusion anesthesia. Hautarzt 2000;51:759-62.

24. McClure JH. Ropivacaine. Br J Anaesth 1996;76:300-7.

25. Pöpping DM, Zahn PK, Van Aken HK, et al. Effectiveness and safety of postoperative pain management: a survey of 18925 consecutive patients between 1998 and 2006 (2nd revision): a database analysis of prospectively raised data. Br J Anaesth 2008;101:832-40. 